\title{
A Low-complexity Successive Detection Method for OFDM Systems over Doubly Selective Channels
}

\author{
Chih-Liang Chen and Sau-Gee Chen
}

\begin{abstract}
For OFDM systems, the time variation of a multipath channel results in inter-carrier interference (ICI). It leads to performance degradations. In reducing the problem, current successive detection methods cost very high computational complexities. Among them, the minimum-mean-squareerror successive detection (MMSE-SD) method has the best performance. This work proposes an improved data detection method with low complexity by integrating the techniques of Newton's iterative matrix inversion method and the MMSE-SD method which considers the effects of signal-to-interference-plusnoise ratio (SINR). In order to efficiently integrate Newton's and MMSE-SD method, we develop an effective scheme with low complexity for generating the initial values required by Newton's method. Based on the new initial value scheme, we are able to simplify the criterion of maximum SINR determination to an equivalent one with lower complexity. As a result, the proposed algorithm has a much lower complexity of $O\left(N^{2}\right)$ than $O\left(N^{3}\right)$ of the MMSE-SD algorithm, where $N$ is the number of subcarriers. Moreover, simulation results in different channel conditions show that performances of the proposed MMSE-SD method are very close to the MMSE-SD method.
\end{abstract}

Index Terms-Orthogonal frequency division multiplexing (OFDM), inter-carrier interference, equalization.

\section{INTRODUCTION}

Since the recent decade, the demand for real-time and highrate multimedia services has been in a rapid growing pace. To satisfy the demand, some advanced broadband communication techniques have been proposed. In particular, the orthogonal frequency division multiplexing (OFDM) technique is widely adopted in current and next-generation communication systems. In an OFDM system, a serial data stream is split into many parallel data streams, each is modulated by its own subcarrier orthogonal to all the other subcarriers. By adding a cyclic prefix (CP) to the beginning of each OFDM symbol, the intersymbol interference (ISI), and intercarrier interference (ICI) caused by delay spreads can be avoided. However, when a channel response is time-varying, the channel variation will introduce ICI and destroy the orthogonality among subcarriers within an OFDM symbol. The ICI effect will seriously degrade

Manuscript received March 01, 2008; revised March 20, and December 31,2008 . The paper was presented in part at the Conference on Software, Telecommunications and Computer Networks (SoftCOM) 2008.

This work is supported in part by the grants NSC 96-2219-E-009-004, MOEA 96-EC-17-A-01-S1-048, and NSC 97-2917-I-009-107, Taiwan. The authors would like to thank the anonymous reviewers for their helpful and constructive comments and suggestions.

Authors are with the Department of Electronics Engineering and Institute of Electronics, National Chiao Tung University, Taiwan (email clchen.nctu@gmail.com,sgchen@cc.nctu.edu.tw). the system performance. As such, the bit-error rate (BER) will increase.

To overcome the ICI problems, several data equalization techniques have been developed, such as the zero-forcing (ZF) and minimum-mean-square-error (MMSE) equalization methods. Unfortunately, both methods require matrix inversion operations which are computationally intensive. Besides, ZF equalization method will result in noise enhancement problem, while MMSE equalization method requires the second-order statistic of a channel. In order to reduce the computational complexity, several simplified methods [1], [2] have been proposed. However, those techniques still have the noise enhancement problem. Specially, the method in [2] applies the wellknown Newton's iterative matrix inversion to approximate the zero-forcing equalization. Still, its performances are limited by the zero-forcing equalization. On the other hand, the method of MMSE equalization with successive detection (MMSE-SD) [3] has good performance but with high computational cost.

To reduce the drawbacks of the mentioned techniques, the objectives of this work are to achieve high performance and simultaneously reduce the computational complexity. Specifically, in order to achieve high performance, MMSE equalization with successive detection (MMSE-SD) method is adopted. To significantly reduce the complexity, this work integrates Newton's matrix inversion method with the MMSESD scheme. Moreover, in order to efficiently apply Newton's matrix inversion method to the MMSE-SD method, a simple but accurate method for generating the initial values for Newton's matrix inversion method is proposed. Furthermore, we design a criterion with a lower computational complexity than the conventional one, in determining the maximum signal-to-interference plus noise ratio (SINR). As a result, the proposed improved MMSE-SD method achieves the goal of high performance and low complexity simultaneously. The rest of this paper is organized as follows. Section II describes the OFDM system model and ICI analysis. In Section III, the proposed improved successive detection method will be introduced. Simulation results of the proposed technique are provided in Section IV. Finally, Section V is the conclusion.

\section{OFDM SYSTEM MODEL AND ICI ANALYSIS}

\section{A. OFDM System Model}

Weinstein [4] suggested that the modulators in the transmitter and the matched filters in the receiver for the OFDM systems can be implemented by IDFT and DFT, respectively. 


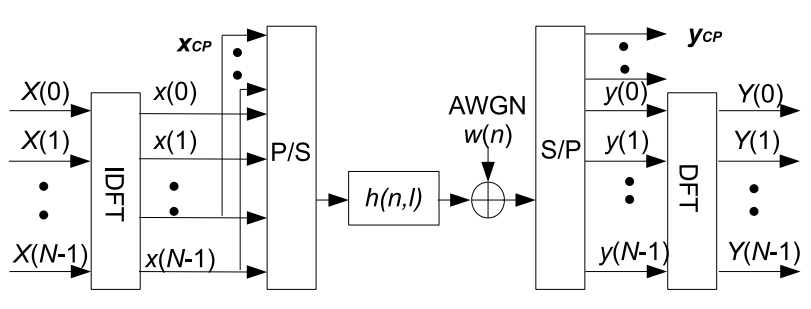

Fig. 1. Discrete-time OFDM system model

Fig. 1 shows the discrete-time OFDM system model considering only one symbol. The modulated signal can be written as

$$
x(n)=\frac{1}{N} \sum_{k=0}^{N-1} X(k) e^{\frac{j 2 \pi k n}{N}},
$$

where $0 \leq n \leq N-1$ and $X(k)$ is the transmitted data at the $k$-th subcarrier. The modulated signal is then preceded with a $\mathrm{CP}$ and delivered through a time-varying multipath fading channel. Therefore, the received signal can be represented as

$$
y(n)=\sum_{l=0}^{L-1} h(n, l) x(n-l)_{N}+w(n)
$$

where $h(n, l)$ is the $l$-th channel path at the time instant $t=n \times t_{s}, t_{s}=\frac{T}{N}$ is the sampling period, $T$ is the symbol duration, $L$ is the number of channel taps, $(\cdot)_{N}$ represents the modulo $N$ operation, and $w(n)$ is the sampled additive white complex Gaussian noise (AWGN) with variance $\sigma^{2}$. The received signal after DFT at the $k$-th subcarrier is

$$
Y(k)=\sum_{n=0}^{N-1} y(n) e^{-\frac{j 2 \pi k n}{N}}
$$

\section{B. ICI Analysis}

According to the system model depicted in Section II-A, the received signal after DFT at the $k$-th subcarrier is obtained by substituting (1) and (2) into (3)

$$
Y(k)=\underbrace{G(k, k) X(k)}_{\text {Desired Signal }}+\underbrace{\sum_{m=0, m \neq k}^{N-1} G(k, m) X(m)}_{I C I}+W(k)
$$

where

$$
\begin{gathered}
G(k, k) \triangleq \frac{1}{N} \sum_{r=0}^{N-1} \sum_{l=0}^{L-1} h(r, l) e^{\frac{-j 2 \pi l k}{N}} \\
G(k, m) \triangleq \frac{1}{N} \sum_{r=0}^{N-1} \sum_{l=0}^{L-1} h(r, l) e^{\frac{j 2 \pi r(m-k)}{N}} e^{\frac{-j 2 \pi l m}{N}}
\end{gathered}
$$

and

$$
W(k)=\sum_{n=0}^{N-1} w(n) e^{-\frac{j 2 \pi k n}{N}}, 0 \leq m, k \leq N-1 .
$$

Thus, the received signal after DFT can be written as the following matrix form

$$
\begin{aligned}
\mathbf{Y} & =\frac{1}{N} \mathbf{Q H} \mathbf{Q}^{H} \mathbf{X}+\mathbf{W} \\
& =\mathbf{G X}+\mathbf{W}
\end{aligned}
$$

where $\mathbf{Y}=[Y(0), \cdots, Y(N-1)]^{T}, \mathbf{X}=[X(0), \cdots, X(N-$ $1)]^{T}, \mathbf{W}=[W(0), \cdots, W(N-1)]^{T}, H(n, m)=h(n,(n-$ $m)_{N}$ ) is the H's element at the $n$-th row and $m$-th column, $\mathrm{Q}$ is an $N$-point DFT matrix with its elements $Q(n, m)=$ $e^{\frac{-j 2 \pi m n}{N}}$, and $\mathbf{G}$ is the channel frequency response matrix with elements $G(k, m)$ at the $k$-row and the $m$-th column.

Since the second term on the right hand side of (4) is not zero when the channel is time-varying, the desired signal $Y(k)$ suffers ICI from all the other subcarriers. However, it can be observed [1] that those ICI terms $\left.G(k, m)\right|_{m \neq k}$ are significant only for $m$ close to $k$. For further ICI analysis, let us first define the time average of channel impulse response, $h(n, l)$, as

$$
h_{a v g}(l) \triangleq \frac{1}{N} \sum_{n=0}^{N-1} h(n, l)
$$

and the variation $\Delta h(n, l)$ of $h(n, l)$ as

$$
\Delta h(n, l) \triangleq h(n, l)-h_{a v g}(l)
$$

By substituting (8) into (5), (5) can be reduced to

$$
G(k, k)=\sum_{l=0}^{L-1} h_{a v g}(l) e^{\frac{-j 2 \pi l k}{N}}
$$

If the channel impulse response is time-invariant within an OFDM symbol, $\Delta h(n, l)$ will be zero and $G(k, m)=0$, for $m \neq k$. In this channel condition, the received signal in (4) only contains the desired signal term. Generally, the channel impulse response will be time-varying, especially for a highly mobile user. Fortunately, the variation of a channel path can be described by a linear function [1] under the conditions of slow fading up to moderately fast fading, particularly when the normalized Doppler frequency (NDF) $f_{n d}$ is roughly less than 0.1 , where NDF is defined as $f_{n d} \triangleq f_{d} T$, and $f_{d}$ is the maximum Doppler frequency. Specifically, the variation of each channel path within an OFDM symbol can be approximated [1], [5], [6] as the following linear function of time index $n$

$\Delta h(n, l)=\left(n-\frac{N-1}{2}\right) \alpha_{l}, 0 \leq n \leq N-1,0 \leq l \leq L-1$,

where $\alpha_{l}$ is the slope of the $l$-th channel path. Given (9), the response of the $l$-th channel path at the $n$-th time instance can be written as

$$
h(n, l)=h_{a v g}(l)+\left(n-\frac{N-1}{2}\right) \alpha_{l}
$$

Therefore, by substituting (12) into (6), (6) can be represented as

$\left.G(k, m)\right|_{m \neq k}=\frac{1}{N} \sum_{r=0}^{N-1} \sum_{l=0}^{L-1}\left(r-\frac{N-1}{2}\right) \alpha_{l} e^{\frac{j 2 \pi r(m-k)}{N}} e^{\frac{-j 2 \pi l m}{N}}$. 
According to (10) and (13), the channel frequency response matrix can be rewritten as

$$
\begin{aligned}
\mathbf{G} & =\frac{1}{N} \mathbf{Q} \mathbf{H} \mathbf{Q}^{H} \\
& =\frac{1}{N} \mathbf{Q}\left(\mathbf{H}_{a v g}+\mathbf{D} \boldsymbol{\alpha}\right) \mathbf{Q}^{H} \\
& =\frac{1}{N}\left(\mathbf{Q} \mathbf{H}_{a v g} \mathbf{Q}^{H}+\mathbf{Q D} \boldsymbol{\alpha} \mathbf{Q}^{H}\right) \\
& =\frac{1}{N}\left(\mathbf{Q} \mathbf{H}_{a v g} \mathbf{Q}^{H}+\mathbf{Q D Q} \mathbf{Q}^{H} \mathbf{Q} \boldsymbol{\alpha} \mathbf{Q}^{H}\right) \\
& =\frac{1}{N}\left(\hat{\mathbf{H}}_{a v g}+\mathbf{Q D D} \mathbf{Q}^{H} \hat{\boldsymbol{\alpha}}\right)
\end{aligned}
$$

where $\mathbf{D}$ is a diagonal matrix and its diagonal elements are $-\frac{N-1}{2}, 1-\frac{N-1}{2}, \cdots, n-\frac{N-1}{2}, \cdots$, and $\frac{N-1}{2}$. Both $\mathbf{H}_{a v g}$ and $\boldsymbol{\alpha}$ are circulant matrices with their elements $H_{\text {avg }}(n, m)=$ $h_{\text {avg }}\left((n-m)_{N}\right)$ and $\alpha(n, m)=\alpha_{(n-m)_{N}}$ at the $n$-th row and the $m$-th column, which contain the static terms and slopes of the channel, respectively. Furthermore, $\hat{\mathbf{H}}_{a v g} \triangleq \mathbf{Q H}_{a v g} \mathbf{Q}^{H}$ and $\hat{\boldsymbol{\alpha}} \triangleq \mathbf{Q}^{H} \boldsymbol{\alpha} \mathbf{Q}^{H}$ are diagonal matrices. Note that (14) will be useful for achieving low complexity of the proposed detection method (as will be described in Section III-E).

\section{Proposed Improved SucCessive Detection METHOD}

First, the MMSE-SD method and Newton's iterative matrix inversion method will be briefly reviewed. Second, an integrated detection method that efficiently takes advantages of these two methods will be proposed. To further reduce complexities while maintain performances, a low-complexity algorithm for the initial inverse matrix and maximum SINR estimation will be introduced.

\section{A. MMSE-SD Method}

The MMSE-SD method [3] can be regarded as an application of MMSE vertical Bell Labs layered space-time (V-BLAST) method. Basically, the whole process can be decomposed into three steps. In the first step, the system equalization matrix is obtained, followed by the second step of determining the maximum SINR subcarrier index and solving the subcarrier data with the equalization matrix. Then, the last step cancels the interferences contributed by the maximum SINR subcarrier, and reconstructs the channel matrix. Eventually, by going back to the first step iteratively, one can obtain the updated equalization matrix, the next maximum SINR subcarrier, and its associated data of the remaining subcarriers.

By canceling the interferences iteratively, the MMSE-SD method will achieve good performance. On the other hand, canceling the interferences iteratively will significantly increase the computational complexity. Hence, it is a trade-off problem between performance and computational complexity. Later, this section will detail a technique that achieves good performance and low computational complexity at the same time.

\section{B. Newton's Iterative Matrix Inversion Method}

For approximating the matrix inversion of a known $N$-by$N$ matrix A, Newton's iterative matrix inversion equation [7] can be written as

$$
\mathbf{U}_{j+1}=\mathbf{U}_{j}\left(2 \mathbf{I}-\mathbf{A} \mathbf{U}_{j}\right), 0 \leq j \leq J
$$

where $\mathbf{I}$ is the identity matrix, $J$ is the number of iteration, which is defined by the system requirement, and $\mathbf{U}_{j}$ is an approximation to $\mathbf{A}^{-1}$ at the $j$-th iteration. Note that (15) requires two matrix multiplications in each iteration. To reduce the complexity, one can expand the iteration equation as

$$
\mathbf{U}_{J}=\sum_{m=1}^{2^{J}} c(J, m)\left\{\left(\mathbf{U}_{0} \mathbf{A}\right)^{m-1}\right\} \mathbf{U}_{0}
$$

where $\mathbf{U}_{0}$ is the initial matrix. Then, the complicated matrix multiplication of $\left(\mathbf{U}_{0} \mathbf{A}\right)^{m-1}$ can be further reduced to vector multiplications. The detailed reduction process will be given in Section III-E. Besides, although there is an increased number of summation terms, the later simulation in Fig. 2 shows that the approximation $\mathbf{U}_{J}$ to $\mathbf{A}^{-1}$ is accurate enough at about the $3^{\text {th }}$ iteration (i.e. $J=3$ ). Hence, the gain in reducing the number of matrix multiplications significantly dominates the cost in the moderately increased number of matrix additions. Besides, in practical realizations, the coefficients $c(J, m)$ can be generated off-line by expanding $\mathbf{U}_{J}$ in terms of $\mathbf{U}_{0}$ and stored in a memory.

\section{Proposed Initial Value Decision Scheme for Newton's Method}

For achieving high performance and low complexity, there are two important issues about how to integrate the successive detection method and Newton's iterative matrix inversion method effectively. One is the way to determine the initial values for Newton's iterative matrix inversion method, which is critically related to both the performance and complexity of Newton's method. The proposed efficient initial values will reduce the complexity without noticeable performance degradation. The other issue is determining the maximum SINR subcarrier index, which is one of the considerably computationconsuming steps in the successive detection method. First, we will propose a low-complexity initial value decision scheme in this subsection. Based on this initial value decision scheme, an accurate and simple criterion for the maximum SINR subcarrier index determination will be proposed in the next subsection.

For low-complexity realization of the initial matrix $\mathbf{U}_{0}$, let $\mathbf{U}_{0}$ be a diagonal matrix as,

$$
\mathbf{U}_{0}=\left[\begin{array}{ccccc}
u_{0} & 0 & \cdots & 0 \\
0 & u_{1} & 0 & \cdots & 0 \\
& 0 & u_{2} & \vdots \\
\vdots & \vdots & & \ddots & 0 \\
0 & \cdots & & 0 & u_{N-1}
\end{array}\right]
$$

By utilizing the minimum squared-error cost function [2], the initial matrix can be determined through the following 
optimization function,

$$
\mathbf{U}_{0}=\underset{\mathbf{U}_{0}}{\arg \min }\left\|\mathbf{R}_{0}\right\|^{2}=\underset{\mathbf{U}_{0}}{\arg \min }\left\|\mathbf{I}-\mathbf{A} \mathbf{U}_{0}\right\|^{2}
$$

where $\left\|\mathbf{R}_{0}\right\|$ means the Frobenius norm of the initial residual matrix $\mathbf{R}_{0}$, and the residual matrix $\mathbf{R}_{j}$ at the $j$-th iteration is defined as $\mathbf{R}_{j}=\mathbf{I}-\mathbf{A} \mathbf{U}_{j}$. Thus, the optimum initial value solutions [2] can be shown to be

$$
u_{k}=\frac{A^{*}(k, k)}{\sum_{m=0}^{N-1}|A(k, m)|^{2}}
$$

where $0 \leq k \leq N-1$ and $A(k, m)$ is the element of $\mathbf{A}$ at the $k$-th row and the $m$-th column.

However, the initial values (19) require significant computation especially when Newton's method is combined with the successive detection method. In order to reduce the complexity of (19), we propose the following simple but accurate method for the generation of the initial values. When applying the Newton's iterative matrix inversion method to MMSE equalization matrix $\left(\mathbf{G}^{H} \mathbf{G}+\sigma^{2} \mathbf{I}_{N}\right)^{-1} \mathbf{G}^{H}$, $\mathbf{A}$ in (16) is equal to $\left(\mathbf{G}^{H} \mathbf{G}+\sigma^{2} \mathbf{I}_{N}\right)$. As analyzed in Appendix A, since $|A(k, k)|^{2}>>\sum_{m=0, m \neq k}^{N-1}|A(k, m)|^{2}$, one can reduce (19) to

$$
\begin{aligned}
u_{k} & =\frac{A^{*}(k, k)}{\sum_{m=0}^{N-1}|A(k, m)|^{2}} \\
& \approx \frac{A^{*}(k, k)}{|A(k, k)|^{2}}=\frac{1}{A(k, k)}
\end{aligned}
$$

Here, for evaluating the performances of (19) and (20) in different NDF conditions, one can define the following performance metric of the normalized mean square error (NMSE) at the $j$-th iteration,

$$
\mathrm{NMSE}_{j}=E\left\{\frac{\sum_{k=0}^{N-1} \sum_{m=0}^{N-1}\left|A^{\prime}(k, m)-U_{j}(k, m)\right|^{2}}{\sum_{k=0}^{N-1} \sum_{m=0}^{N-1}\left|A^{\prime}(k, m)\right|^{2}}\right\}
$$

where $E\{$.$\} is the expectation value operator, A^{\prime}(k, m)$ and $U_{j}(k, m)$ are the elements of $\mathbf{A}^{-1}$ and $\mathbf{U}_{j}$, respectively, at the $k$-th row and the $m$-th column, and $0 \leq j \leq J$. NMSE represents the normalized difference between the ideal inverse matrix and output of Newton's iterative matrix inversion method. By applying (19) and (20) to Newton's method, one can evaluate the NMSE performances due to these two initial values. According to Fig. 3, the NMSE curves of (19) and (20) virtually overlap. However, (20) has a much lower computational complexity than (19). Especially, this outcome still holds even in high NDF conditions. Also shown is that both initial value estimations give very small errors around $10^{-5}$ even after the second iteration, and converge to around $10^{-30}$ after the $5^{\text {th }}$ iteration.

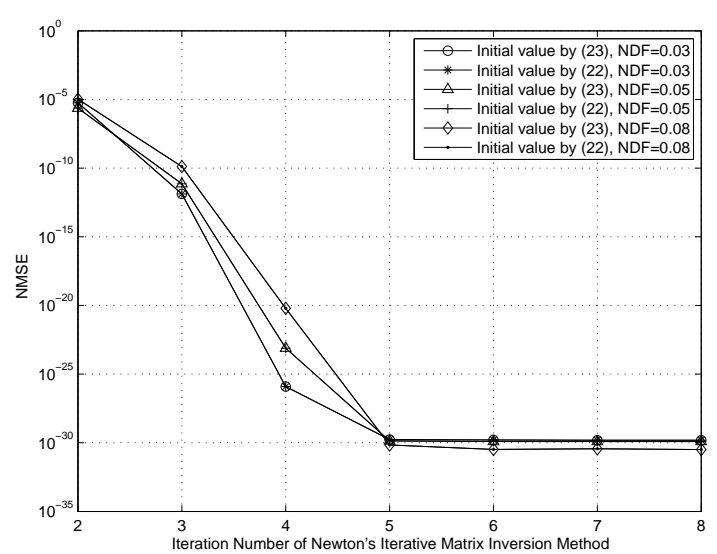

Fig. 2. NMSEs of (22) and (23) versus iteration number in different NDFs

\section{Proposed Criterion for Maximum SINR Determination}

Next, we need to determine the maximum SINR subcarrier index based on the mentioned initial matrix. Note that here we apply Newton's iterative matrix inversion method to the evaluation of $\left(\mathbf{G}^{H} \mathbf{G}+\sigma^{2} \mathbf{I}_{N}\right)^{-1}$, so that the approximate MMSE equalization matrix at the $J$-th iteration is

$$
\left(\mathbf{G}^{H} \mathbf{G}+\sigma^{2} \mathbf{I}_{N}\right)^{-1} \mathbf{G}^{H} \approx \mathbf{U}_{J} \mathbf{G}^{H}
$$

Then, the estimated MMSE $\tilde{\mathbf{X}}$ of $\mathbf{X}$ in (7) at the $J$-th iteration is

$$
\begin{aligned}
\tilde{\mathbf{X}} & =\mathbf{U}_{J} \mathbf{G}^{H} \mathbf{Y} \\
& =\mathbf{U}_{J} \mathbf{G}^{H} \mathbf{G} \mathbf{X}+\mathbf{U}_{J} \mathbf{G}^{H} \mathbf{W}
\end{aligned}
$$

Moreover, to achieve high performance, successive detection method is adopted. For successive detection, the conventional criterion for determining the maximum SINR subcarrier index [3] is given as

$$
\underset{k}{\operatorname{argmax} S I N R}=\underset{k}{\operatorname{argmax}} \frac{\left|<\hat{\mathbf{u}}_{J, k}, \mathbf{g}_{k}>\right|^{2}}{\sum_{\substack{m \neq k \\ m=0}}^{N-1} k \hat{\mathbf{u}}_{J, k}, \mathbf{g}_{m}>\left.\right|^{2}+\sigma^{2}\left\|\hat{\mathbf{u}}_{J, k}\right\|^{2}}
$$

where $\hat{\mathbf{u}}_{J, k}$ is the $k$-th row vector of the equalizer matrix $\mathbf{U}_{J} \mathbf{G}^{H}, \mathbf{g}_{k}$ is the $k$-th column vector of $\mathbf{G}$, and $<.>$ is the inner product operator.

Clearly, (23) is very complicated. However, since the accumulation term of the denominator $\sum_{m \neq k, m=0}^{N-1} k \hat{\mathbf{u}}_{J, k}, \mathbf{g}_{m}>\left.\right|^{2}$ is expectedly smaller than the noise term $\sigma^{2}\left\|\hat{\mathbf{u}}_{J, k}\right\|^{2}$ in reasonable SNR conditions [12] and the inner product $\left\langle\hat{\mathbf{u}}_{J, k}, \mathbf{g}_{k}\right\rangle$ is close to unity, (23) can be simplified as

$$
\begin{aligned}
\underset{k}{\arg \max } \operatorname{SINR} & \approx \underset{k}{\arg \max } \frac{\left|<\hat{\mathbf{u}}_{J, k}, \mathbf{g}_{k}>\right|^{2}}{\sigma^{2}\left\|\hat{\mathbf{u}}_{J, k}\right\|^{2}} \\
& \approx \underset{k}{\arg \max } \frac{1}{\sigma^{2}\left\|\hat{\mathbf{u}}_{J, k}\right\|^{2}} \\
& \approx \underset{k}{\arg \min }\left\|\hat{\mathbf{u}}_{J, k}\right\|^{2}
\end{aligned}
$$

Since according to (18), $\mathbf{U}_{0}$ is the closet diagonal matrix to the inverse matrix of $\mathbf{A}, \mathbf{U}_{0} \mathbf{A}$ can be reasonably assumed to 
be close to an identity matrix (because the diagonal elements of $\mathbf{A}$ here are much larger than the off-diagonal elements), and (16) can be approximated as

$$
\begin{aligned}
\mathbf{U}_{J} & =\sum_{m=1}^{2^{J}} c(J, m)\left\{\left(\mathbf{U}_{0} \mathbf{A}\right)^{m-1}\right\} \mathbf{U}_{0} \\
& \approx \sum_{m=1}^{2^{J}} c(J, m) \mathbf{I}^{m-1} \mathbf{U}_{0} \\
& \approx \sum_{m=1}^{2^{J}} c(J, m) \mathbf{U}_{0}
\end{aligned}
$$

Finally, by substituting (25) for $\mathbf{U}_{J}$ in $\mathbf{U}_{J} \mathbf{G}^{H}$, one has the following simplified optimization function

$$
\begin{aligned}
\underset{k}{\arg \max S I N R} & \approx \underset{k}{\arg \min }\left\|\hat{\mathbf{u}}_{J, k}\right\|^{2} \\
& \approx \underset{k}{\arg \min }\left\|\hat{\mathbf{u}}_{0, k}\right\|^{2} \\
& \approx \underset{k}{\arg \min }\left|u_{k} \times G(k, k)\right|^{2}
\end{aligned}
$$

which has a much lower complexity than (23).

\section{E. Proposed Improved Sub-block MMSE Successive Detection Method}

In fact, in (16), $\left(\mathbf{U}_{0} \mathbf{A}\right)^{m-1}$ still requires a lot of computation. Besides, in order to obtain the equalization matrix $\left(\mathbf{G}^{H} \mathbf{G}+\sigma^{2} \mathbf{I}_{N}\right)^{-1} \mathbf{G}^{H}$ for MMSE equalizer, the result of Newton's iterative matrix inversion method still has to perform matrix multiplication with $\mathbf{G}^{H}$.

This work will utilize the channel responses in time domain instead of the channel frequency responses in frequency domain to significantly reduce the computational complexity as follows. First, we substitute (16) into (22) and solve the output $\tilde{\mathbf{X}}$ of the linear MMSE equalizer as

$$
\begin{aligned}
\tilde{\mathbf{X}} & =\mathbf{U}_{J} \mathbf{G}^{H} \mathbf{Y} \\
& =\sum_{m=1}^{2^{J}} c(J, m)\left\{\left(\mathbf{U}_{0} \mathbf{A}\right)^{m-1}\right\} \mathbf{U}_{0} \mathbf{G}^{H} \mathbf{Y} \\
& =\sum_{m=1}^{2^{J}} c(J, m) \mathbf{s}_{m}
\end{aligned}
$$

where $\mathbf{s}_{m}$ is defined as

$$
\mathbf{s}_{m} \triangleq\left\{\left(\mathbf{U}_{0} \mathbf{A}\right)^{m-1}\right\} \mathbf{U}_{0} \mathbf{G}^{H} \mathbf{Y}
$$

and note that $\mathbf{A}=\left(\mathbf{G}^{\mathbf{H}} \mathbf{G}+\sigma^{2} \mathbf{I}_{\mathbf{N}}\right)$.

Thus, $\tilde{\mathbf{X}}$ can be obtained by summing all $c(J, m) \mathbf{s}_{m}$ terms, where $\mathbf{s}_{m}$ can be recursively calculated by the equation

$$
\mathbf{s}_{m}=\left\{\mathbf{U}_{0}\left(\mathbf{G}^{H} \mathbf{G}+\sigma^{2} \mathbf{I}_{N}\right)\right\} \mathbf{s}_{m-1}
$$

Relying on (28), one can simplify all the matrix operations of (27) to vector operations except $\mathbf{G}^{H} \mathbf{G}$ in $\mathbf{A}$. The $\mathbf{G}^{H} \mathbf{G}$ term can be further expanded by substituting (14), $\mathbf{G}=\frac{1}{N}\left(\hat{\mathbf{H}}_{a v g}+\right.$ $\mathbf{Q D Q} \mathbf{Q}^{H} \hat{\boldsymbol{\alpha}}$ ), into $\mathbf{G}^{H} \mathbf{G}$ as

$$
\begin{aligned}
\mathbf{G}^{H} \mathbf{G}= & \frac{1}{N^{2}}\left\{\hat{\mathbf{H}}_{a v g}^{H} \hat{\mathbf{H}}_{a v g}+\left(\mathbf{Q D} \mathbf{Q}^{H} \hat{\boldsymbol{\alpha}}\right)^{H} \hat{\mathbf{H}}_{a v g}\right. \\
& \left.+\hat{\mathbf{H}}_{a v g}^{H}\left(\mathbf{Q} \mathbf{D} \mathbf{Q}^{H} \hat{\boldsymbol{\alpha}}\right)+\hat{\boldsymbol{\alpha}}^{H} \mathbf{Q} \mathbf{D}^{H} \mathbf{D} \mathbf{Q}^{H} \hat{\boldsymbol{\alpha}}\right\}
\end{aligned}
$$

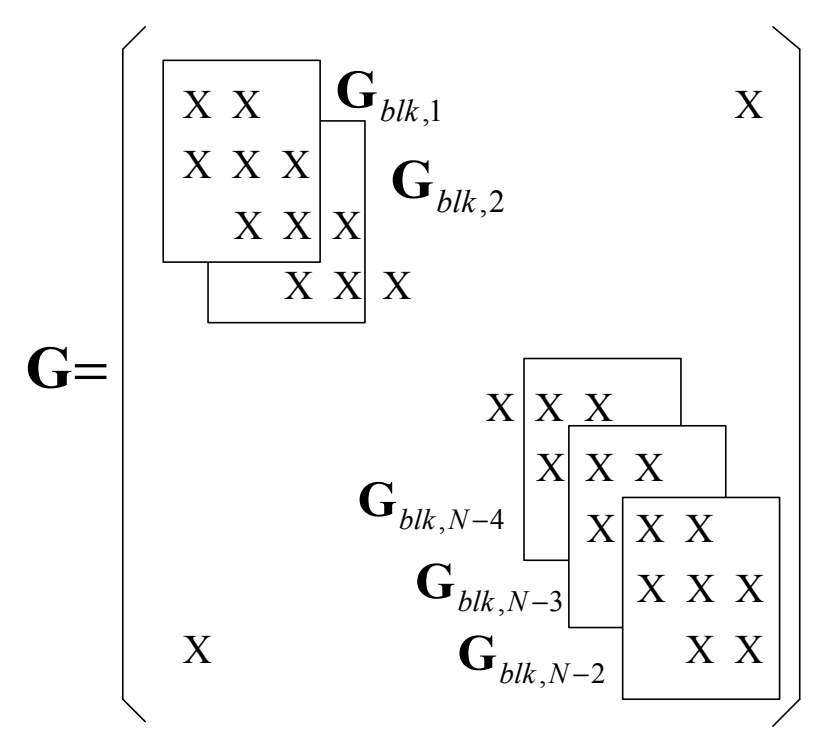

Fig. 3. An example $\mathbf{G}$ of matrix simplification and partitioning, $q=1$

Note that $\mathbf{Q D Q} \mathbf{Q}^{H}$ and $\mathbf{Q} \mathbf{D}^{H} \mathbf{D} \mathbf{Q}^{H}$ can be obtained in advance. Since $\hat{\mathbf{H}}_{\text {avg }}$ and $\hat{\boldsymbol{\alpha}}$ are diagonal matrices which contain the time-domain channel information, (29) can be realized with low complexity. Thus, based on the above discussion, $\tilde{\mathbf{X}}$ can be obtained with low computational complexity.

In fact, according to the ICI discussion in Section II-B, only a few ICI elements close to the diagonal elements in $\mathbf{G}$ are significant. By considering only those central diagonal ICI terms, one can separate matrix $\mathbf{G}$ into $N(2 q+1)$-by- $(2 q+1)$ sub-matrices as (30), where $0 \leq k \leq N-1$. We assume that there is no ICI effect needed to be considered except the $2 q$ most adjacent subcarriers to the central subcarrier. As a result, each $\mathbf{G}_{b l k, k}$ is a $(2 q+1)$-by- $(2 q+1)$ matrix. Fig. 3 gives an example of $q=1$.

Through the sub-matrix partition procedure, the linear MMSE equalizer can be modified from one $N$-by- $N$ matrix to $N(2 q+1)$-by- $(2 q+1)$ matrices as follows.

$$
\mathbf{U}_{J, b l k, k} \mathbf{G}_{b l k, k}^{H}=\left(\mathbf{G}_{b l k, k}^{H} \mathbf{G}_{b l k, k}+\sigma^{2} \mathbf{I}_{2 q+1}\right)^{-1} \mathbf{G}_{b l k, k}^{H},
$$

where $0 \leq k \leq N-1$. Thus, the equalized data vector $\tilde{\mathbf{X}}_{b l k, k}$ corresponding to the $k$-th subcarrier in (27) can be rewritten as

$$
\tilde{\mathbf{X}}_{b l k, k}=\sum_{m=1}^{2^{J}} c(J, m) \mathbf{s}_{m, b l k, k}
$$

where $\mathbf{s}_{m, b l k, k}$ can be calculated recursively by

$$
\mathbf{s}_{m, b l k, k}=\left\{\mathbf{U}_{0, b l k, k}\left(\mathbf{G}_{b l k, k}^{H} \mathbf{G}_{b l k, k}+\sigma^{2} \mathbf{I}_{(2 q+1)}\right)\right\} \mathbf{s}_{m-1, b l k, k}
$$

Although there are $N$ equalized data vectors $\tilde{\mathbf{X}}_{b l k, k}, 0 \leq$ $k \leq N-1$, to be calculated according to (32), only the center scalar element $\tilde{X}_{b l k, k}(q+1)$ (shown below) of each $\tilde{\mathbf{X}}_{b l k, k}$ vector is needed by the proposed successive detection method.

$$
\tilde{X}_{b l k, k}(q+1)=\sum_{m=1}^{2^{J}} c(J, m) s_{m, b l k, k}(q+1),
$$




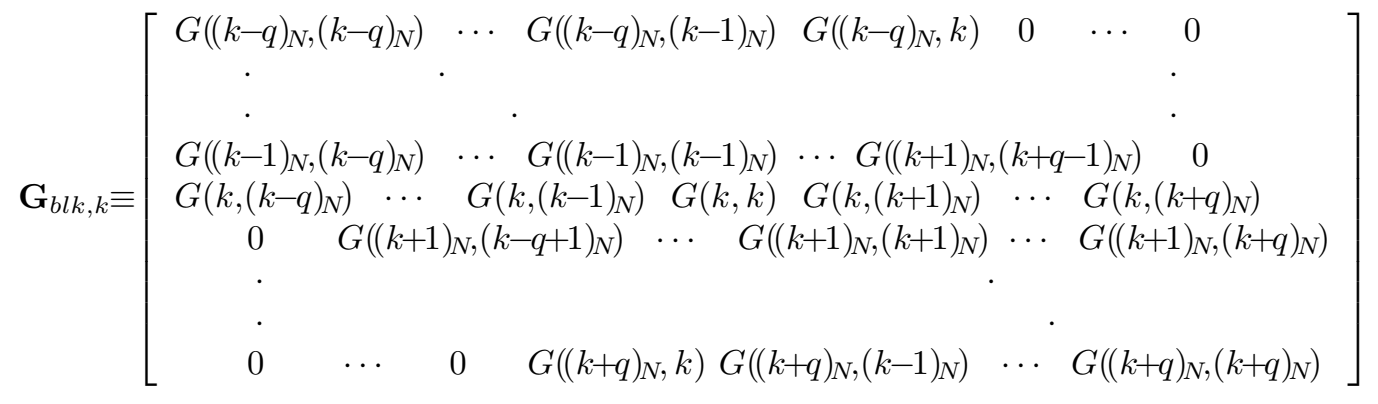

As a result, by utilizing the sub-matrix approximation method, the improved sub-block MMSE detection method (ISBMMSE-SD) can reduce the complexity of updating $\mathbf{s}_{m}$ with $N$-dimensional operations to that of updating $N$ scalar components $s_{m, b l k, k}(q+1), 0 \leq k \leq N-1$, which are the $(q+1)$-th (center) components of vector $\mathbf{s}_{m, b l k, k}$.

As discussed above, the successive detection process will be performed according to the subcarrier order from high SINR to low SINR, which is determined by (26), while (32) will be applied to the equalization process. The complete procedures of the ISB-MMSE-SD method are summarized below.

Step 1: Set $n=1$ as the initial value of the subcarrier counter. Also set a proper Newton's iteration number $J$.

Step 2: Determine the maximum SINR subcarrier index through the proposed criterion (26):

$$
i_{1}=\underset{k}{\arg \min }\left|u_{b l k, k} \times G(k, k)\right|^{2}
$$

where $0 \leq k \leq N-1$ and $u_{b l k, k}$ is the element of $\mathbf{U}_{0, b l k, k}$ at the $(q+1)$-th row and the $(q+1)$-th column, which is obtained by using (20) (assuming $\mathbf{A}=\mathbf{G}_{b l k, k}^{H} \mathbf{G}_{b l k, k}+$ $\sigma^{2} \mathbf{I}_{(2 q+1)}$.

Step 3: Perform the proposed Newton's-method-based MMSE equalization process for the subblock on the selected subcarrier $i_{n}$ :

$$
\tilde{X}_{b l k, i_{n}}(q+1)=\sum_{m=1}^{2^{J}} c(J, m) s_{m, b l k, i_{n}}(q+1)
$$

where $\tilde{X}_{b l k, i_{n}}(q+1)$ and $s_{m, b l k, i_{n}}(q+1)$ are the $(q+$ 1)-th data of the selected subcarrier in $\tilde{\mathbf{X}}_{b l k, i_{n}}$ and $\mathbf{s}_{m, b l k, i_{n}}$, respectively. Based on Newton's method, note that $s_{m, b l k, i_{n}}(q+1), 1 \leq m \leq 2^{J}$, can be obtained through taking out the center element of $\mathbf{s}_{m, b l k, i_{n}}$ in (33), iteratively. Step 4: Perform the data decision for the data at the selected subcarrier $i_{n}$ :

$$
\left.\hat{X}\left(i_{n}\right)=\operatorname{slice}\left(\tilde{X}_{b l k, i_{n}}(q+1)\right)\right)
$$

Step 5: Cancel the interferences from the selected subcarrier $i_{n}$ :

$$
\mathbf{Y}=\mathbf{Y}-\mathbf{g}_{i_{n}} \hat{X}\left(i_{n}\right)
$$

where $\mathbf{g}_{i_{n}}$ is the $i_{n}$-th column vector of $\mathbf{G}$.

Step 6: Reconstruct the new channel matrix by padding a $N$-by-1 zero vector at $i_{n}$-th column of $\mathbf{G}$ :

$$
\mathbf{G}=\left[\mathbf{g}_{0}, \cdots, \mathbf{g}_{i_{n}-1}, \mathbf{0}, \mathbf{g}_{i_{n}+1}, \cdots, \mathbf{g}_{N-1}\right]
$$

Step 7: Determine the maximum SINR subcarrier of all the remaining subcarriers:

$$
i_{n+1}=\underset{k \notin\left\{i_{1} \cdots i_{n}\right\}}{\arg \min }\left|u_{b l k, k} \times G(k, k)\right|^{2}
$$

Step 8: Increase the subcarrier counter by one, i.e. $n=n+1$. If $n \neq N$, go to Step 3; else, the end of the whole process. As shown above, the proposed method relies on the timedomain channel knowledge. There are many time-domain channel estimation techniques in literature can be utilized here. For example, the one in [11] has good performances. Next, the performance of the proposed ISB-MMSE-SD method will be evaluated.

\section{PERformances AnAlysis}

\section{A. Complexity Analysis}

Fig. 4 shows the structure of the proposed improved subblock MMSE successive detection method. As shown, there are seven main function blocks for the following demonstration and the detailed complexity analysis.

- Block A: There are two kinds of modes in block A. One is the initial mode; the other is the iteration mode. At the beginning, block A requires $4 N^{2}$ real multiplication operations and $3 N^{2}$ real addition operations in the initial mode. Then, block A will deliver the result of $\left(\mathbf{G}^{H} \mathbf{G}+\right.$ $\left.\sigma^{2} \mathbf{I}\right)$ to the next block. After that, block A turns into the iteration mode. In the iteration mode, block A just needs to deliver $\sigma^{2}$ to the next block for updating the previous selected subcarrier in block B without any computation.

- Block B: Similar to block A, block B has two modes. In the initial mode, block B needs to perform $N$ division operations for determining the highest SINR subcarrier. After the initial mode, block B updates $u_{b l k, i}$ with one real division operation, where $i$ is the selected subcarrier corresponding to step 7 of the proposed ISB-MMSE-SD method.

- Block C: In order to determine the maximum SINR subcarrier, block $\mathrm{C}$ only requires $2 N$ multiplication operations in the initial mode. After the initial mode, block $\mathrm{C}$ just needs to select the among the remaining subcarriers without any computation according to the result coming from the initial mode.

- Block D: In order to obtain the initial value of the accumulation terms in $\mathbf{s}_{1, b l k, k}$ block D requires $\left[(2 q+1)^{2}+\right.$ $16(2 q+1)]$ real multiplication and $\left[(2 q+1)^{2}+8(2 q+1)\right]$ real addition operations. 


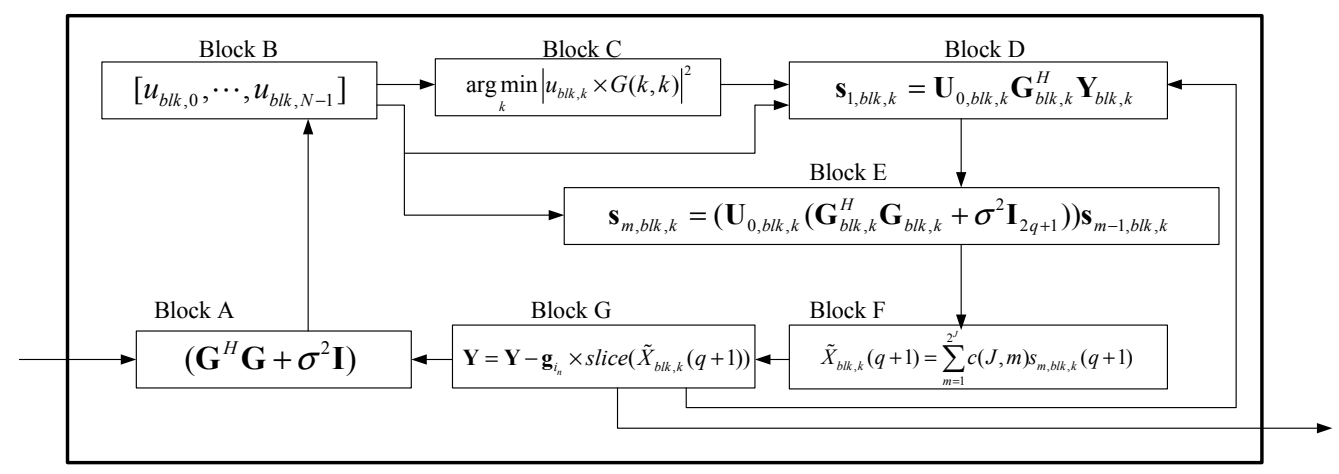

Fig. 4. Structure of ISB-MMSE-SD method for complexity analysis

TABLE I

COMPLEXITY COMPARISON

\begin{tabular}{|c|c|c|c|}
\hline Methods & $\begin{array}{l}\text { Number of } \\
\text { Additions }\end{array}$ & $\begin{array}{c}\text { Number of } \\
\text { Multiplications }\end{array}$ & $\begin{array}{c}\text { Number of } \\
\text { Divisions }\end{array}$ \\
\hline Zero-forcing & $\begin{array}{c}11 N^{3}-3 N^{2} \\
-4 N\end{array}$ & $\begin{array}{c}12 N^{3}+6 N^{2} \\
-10 N\end{array}$ & $2 N^{2}-2 N$ \\
\hline MMSE-SD [3] & $O\left(N^{3}\right)$ & $O\left(N^{3}\right)$ & $O\left(N^{2}\right)$ \\
\hline $\begin{array}{c}\text { Proposed } \\
\text { ISB-MMSE-SD }\end{array}$ & $\begin{array}{c}8 N^{2} \\
+\left\{\left(12 \times 2^{J}-11\right)\right. \\
\times(2 q+1)^{2} \\
+\left(4 \times 2^{J}+4\right) \\
\times(2 q+1) \\
\left.+\left(6 \times 2^{J}+4\right)\right\} N\end{array}$ & $\begin{array}{c}8 N^{2} \\
+\left\{\left(12 \times 2^{J}-11\right)\right. \\
\times(2 q+1)^{2} \\
+\left(16 \times 2^{J}\right) \\
\times(2 q+1) \\
\left.+\left(10 \times 2^{J}+6\right)\right\} N\end{array}$ & $N^{2}+N$ \\
\hline
\end{tabular}

- Block E: For obtaining the rest of the accumulation terms in (46), block E requires $\left[\left(3 \times 2^{J}-3\right) \times(2 q+1)^{2}+(4 \times\right.$ $\left.\left.2^{J}-4\right) \times(2 q+1)+\left(2 \times 2^{J}-2\right)\right]$ multiplication and $\left[\left(3 \times 2^{J}-3\right) \times(2 q+1)^{2}-\left(2 \times 2^{J}-2\right) \times(2 q+1)\right]$ addition operations. Note that we only need to obtain the elements of the selected subcarrier in (46).

- Block F: Clearly, the equalization and data detection require $2 \times 2^{J}$ real multiplication and $\left(2^{J}-1\right)$ addition operations.

- Block G: The ICI cancellation here needs $N$ multiplication and $N$ addition operations.

Note that all the mentioned operations are done on complex numbers, unless indicated specifically. Due to the successive detection, all the functions in these blocks are performed $N$ times. However, block A, B, and $\mathrm{C}$ will be turned into the iteration mode after that the highest SINR subcarrier index has been detected.

The total complexity of the proposed ISB-MMSE-SD method is summarized in Table I. Also given are the complexities of the MMSE-SD method and the zero-forcing detection method, where $O(\cdot)$ means "order of". In the MMSE-SD method, the most computation-consuming steps are in iteratively determining the highest SINR subcarrier and performing equalization operations. We have greatly simplified the process by applying the result of (26) and (32) to the ISB-MMSE-SD

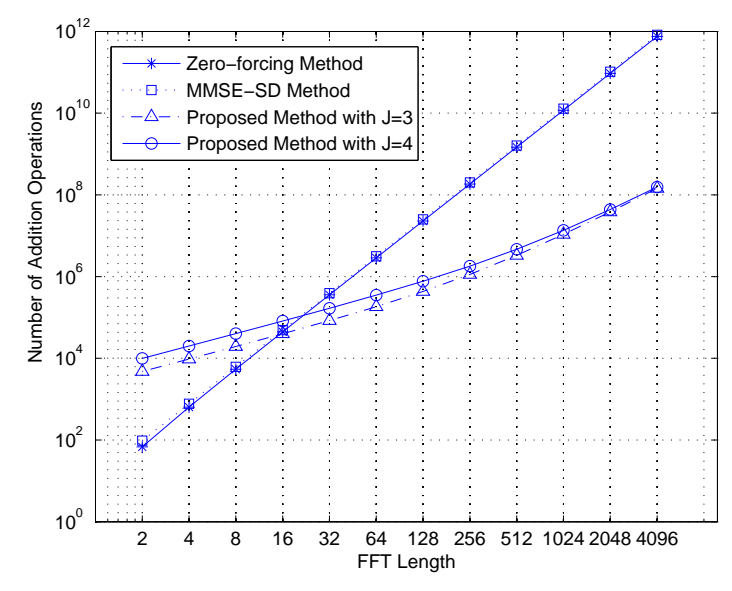

Fig. 5. Number of addition operations versus FFT length

method.

Besides, Fig. 5, Fig. 6, and Fig. 7 show the complexities of addition, multiplication, and division operations for three different methods under various values of FFT lengths $N$, respectively. As shown in Fig. 5 and Fig. 6, when the FFT length is larger than 16, the proposed ISB-MMSE-SD method has fewer addition and multiplication operations than the conventional methods. Similarly, as shown in Fig. 7, ISBMMSE-SD method has fewer division operations than the conventional methods. Thus, the proposed method has much lower complexity than the compared methods, and meantime achieves high performance as verified below.

\section{B. Simulation Results}

Here, we simulate an OFDM system assuming $N=64$ subcarriers, CP length $L_{c p}=16$. The adopted modulations are QPSK and 16-QAM. The simulation channel is a Rayleigh fading channel described in [8]. The power delay profile is chosen as the "Vehicular B" highly selective channel model defined by ETSI for the evaluation of UMTS radio interface proposals [9]. Since this channel has a large delay spread, it is frequency selective. Furthermore, the bit error rate (BER) performance of the proposed detection algorithms are compared with those of the zero-forcing and the MMSE-SD schemes [3]. Fig. 8 and Fig. 9 show the BER simulation results of all the 


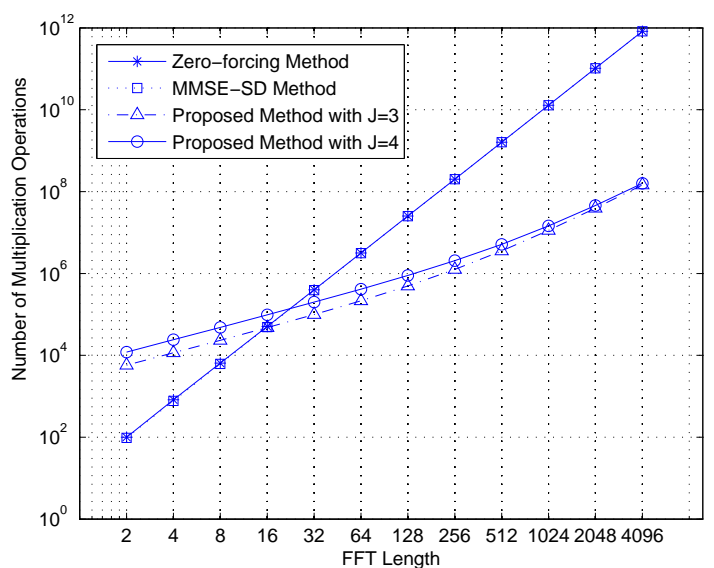

Fig. 6. Number of multiplication operations versus FFT length

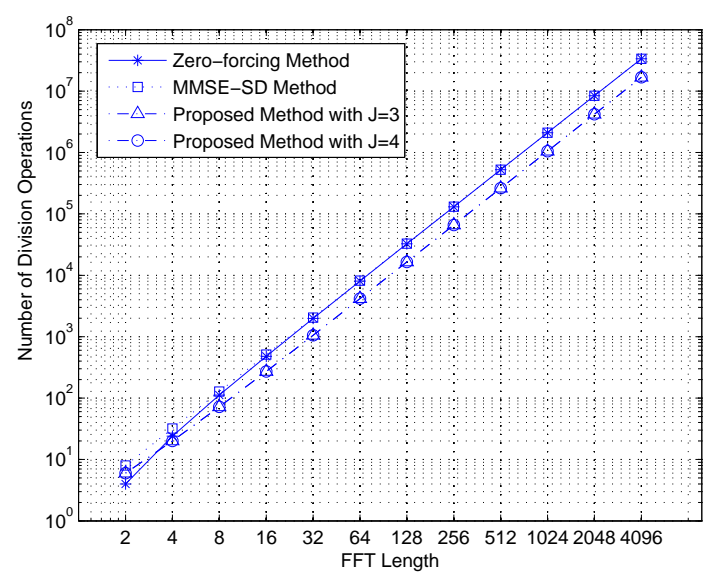

Fig. 7. Number of division operations versus FFT length

mentioned methods, under two different normalized Doppler frequency settings of $f_{n d}=0.05$ and $f_{n d}=0.08$.

Since the detection results are almost independent of the number $N$ of total subcarriers, we did not run simulation assuming higher $N$ than 64 in the manuscript (as we expect that the results would be the same as the lower $N$ cases). The reason why is due to the fact that the channel variation is independent of the total number of subcarriers [8]. Moreover, although the inter-carrier interference (ICI) term in (4) is dependent on $N$, it is only dominated by a few ICI terms coming from the adjacent subcarriers closet to the $k$-th subcarrier. According to simulation results, even when the number $q$ of the considered adjacent subcarriers are as small as 2, the simulated performance has already been very close to the optimal results (which consider all the ICI subcarriers). For $q$ greater than 2, the results are even better and virtually the same as the optimal results. That means the effect of parameter $N$ 's magnitude on the simulation results is basically indiscernible for $N$ greater than a single-digit value. As such, it is expected that the same simulation results would be generated for subcarrier numbers higher than 64 , and the simulation results with 64 subcarriers can also be

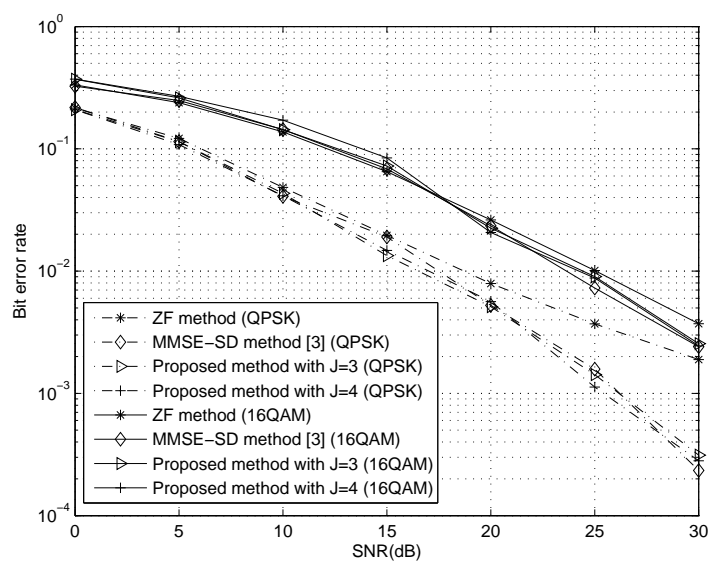

Fig. 8. BER performance comparison at $\mathrm{NDF}=0.05$

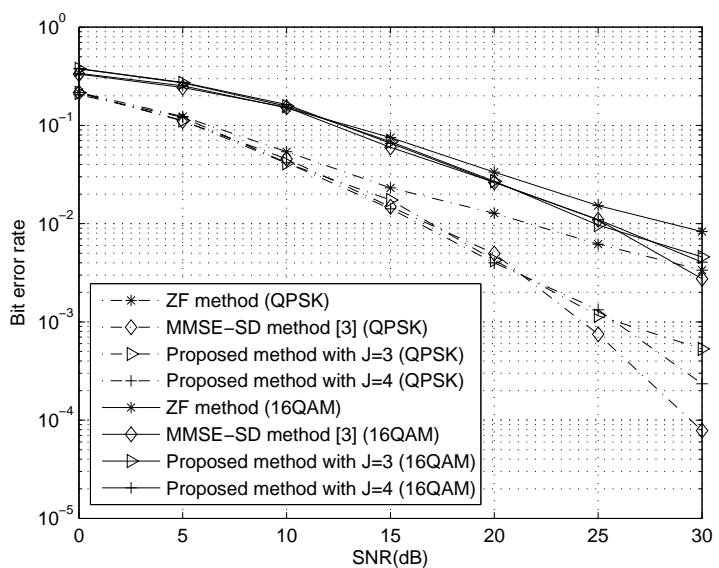

Fig. 9. BER performance comparison at $\mathrm{NDF}=0.08$

applied to other systems with more subcarriers, such as IEEE $802.11 \mathrm{n} / 16 \mathrm{e}$ and DVB-T/H.

As shown in the figures, the proposed ISB-MMSE-SD method has better performance than the zero-forcing (ZF) method regardless of the adopted modulation, with lower complexity, in both simulation environments. On the other hand, the ISB-MMSE-SD method only considers the 4 most adjacent neighboring subcarriers, i.e. $q=2$. Clearly, the performance of the ISB-MMSE-SD method approaches that of the MMSE-SD method very closely, but requires much less complexity than the MMSE-SD method. Moreover, with 16-QAM modulation, Fig. 8 and Fig. 9 also show that the performance of the proposed ISB-MMSE-SD method is closer to the MMSESD method than those of ISB-MMSE-SD method with QPSK modulation.

\section{CONClusion}

This work presents an improved sub-block MMSE successive detection method with low computational complexity. Especially, simulation results under the conditions of simultaneous presence of time and frequency selective show better performance than the zero-forcing approach and close perfor- 
mance to the MMSE-SD method, but with much lower computational complexity than both methods. Hence, the proposed improved method can be effectively applied to OFDM systems in time-varying and multi-path Rayleigh fading channels such as WLAN, DVB-H and WiMax systems, and etc..

$$
\text { Proof of }|A(k, k)|^{2}>>\left.\sum_{m=0, m \neq k}^{\text {ApPendix }}\right|_{N-1} ^{\mathrm{A}-1}|A(k, m)|^{2}
$$

Since $\mathbf{A}=\left(\mathbf{G}^{H} \mathbf{G}+\sigma^{2} \mathbf{I}_{N}\right)$, the elements in $\mathbf{A}$ are

$$
A(k, m)=\sum_{s=0}^{N-1} G^{*}(s, k) G(s, m)+\sigma^{2}
$$

Here, these elements are classified into two groups. One is the group of diagonal elements, called the diagonal group. The other is the group of the remaining non-diagonal elements, called the non-diagonal group. As discussed in Section II-B and shown in Fig. 2,

$$
|G(k, k)|^{2}>>\sum_{m=0, m \neq k}^{N-1}|G(k, m)|^{2},
$$

at reasonable SNR, and the square absolute values of those two group are separately given by

$$
\left\{\begin{array}{l}
\text { diagonal group: } \\
|A(k, k)|^{2} \approx|G(k, k)|^{4} \\
\text { non-diagonal group: } \\
|A(k, m)|_{k \neq m}^{2} \approx \sum_{s=0, m \neq k}^{N-1}|G(s, k)|^{2}|G(s, m)|^{2}
\end{array}\right.
$$

The comparison will start at summing square absolute values of the non-diagonal group as follows,

$$
\begin{aligned}
\sum_{\substack{m=0 \\
m \neq k}}^{N-1}|A(k, m)|^{2} & \approx \sum_{\substack{m=0 \\
m \neq k}}^{N-1} \sum_{\substack{s=0 \\
k \neq m}}^{N-1}|G(s, k)|^{2}|G(s, m)|^{2} \\
& \approx \sum_{\substack{s=0 \\
k \neq m}}^{N-1}|G(s, k)|^{2} \sum_{\substack{m=0 \\
m \neq k}}^{N-1}|G(s, m)|^{2}
\end{aligned}
$$

Again, because of (36), (38) can be further derived as

$$
\begin{aligned}
\sum_{\substack{m=0 \\
m \neq k}}^{N-1}|A(k, m)|^{2} & <<\sum_{\substack{s=0 \\
k \neq m}}^{N-1}|G(s, k)|^{2}|G(s, s)|^{2} \\
& <<|G(k, k)|^{4}
\end{aligned}
$$

By combining (37) and (39), one can obtain the following relationship of the elements in the initial matrix for Newton's iterative inversion method.

$$
|A(k, k)|^{2}>>\sum_{\substack{m=0 \\ m \neq k}}^{N-1}|A(k, m)|^{2}
$$

\section{ACKNOWLEDGMENT}

The authors would like to thank the anonymous reviewers for their helpful and constructive comments and suggestions. This work is supported in part by the grants NSC 96-2219-E009-004 and MOEA 96-EC-17-A-01-S1-048, Taiwan.

\section{REFERENCES}

[1] W. G. Jeon, K. H. Chang, and Y. S. Cho, ”An Equalization Technique for Orthogonal Frequency-Division Multiplexing Systems in Time-Variant Multipath Channels," IEEE Trans. Commun., vol. 47, no.1, pp.27-32, Jan. 1999.

[2] C. Y. Hsu and W. R. Wu, "A low-complexity ICI mitigation method for high-speed mobile OFDM systems," in Proc. IEEE Circuits and Systems (ISCAS) 2006, May 2006, pp. 21-24.

[3] Y. S. Choi, P. J. Voltz, and F. A. Cassara, "On channel estimation and detection for multicarrier signals in fast and selective rayleigh fading channels," IEEE Trans. Commun., vol. 49, pp. 1375-1387, Aug. 2001.

[4] S. B. Weinstein and P. M. Ebert, "Data transmission by frequency-division multiplexing using the discrete Fourier transform," IEEE Trans. Commun., vol. COM-19, pp. 628-634, Oct. 1971.

[5] Y. Mostofi and D. C. Cox, "ICI Mitigation for Pilot-Aided OFDM Mobile Systems," IEEE Trans. Wireless Commun., vol. 4, no. 2, pp. 765-774, Mar. 2005.

[6] Y. H. Yeh and S. G. Chen, "Reduction of Doppler-induced ICI by interference prediction," in Proc. IEEE Personal, Indoor and Mobile Radio Communications (PIMRC) 2004, Sept. 2004, vol. 1, pp. 653-657.

[7] A. S. Householder, The Theory of Matrix in Numerical Analysis. New York: Dover Publications, 1964, pp. 94-98.

[8] Y. R. Zheng and C. Xiao, "Simulation Models With Correct Statistical Properties for Rayleigh Fading Channels," IEEE Trans. Commun., vol. 51, no. 6, pp. 920-928, June, 2003.

[9] ETSI TR 101112 V3.2.0, "Universal Mobile Telecommunications System (UMTS); Selection Procedures for the Choice of Radio Transmission Technologies of the UMTS (UMTS 30.03 version 3.2.0)," ETSI, Apr. 1998.

[10] C. L. Chen and S. G. Chen, "Low-complexity Successive Detection Method for OFDM Systems over Doubly Selective Channels," in Proc. IEEE Software, Telecommunications and Computer Networks (SoftCOM) 2007, Sep. 2007.

[11] D. K. Borah and B. D. Hart, "Frequency-selective fading channel estimation with a polynomial time-varying channel model," IEEE Trans. Commun., vol. 47, no. 6, pp. 862-873, June, 1999.

[12] D. Tse and P. Viswanath, Fundamentals of Wireless Communication, Cambridge University Press, 2005, pp. 356-362.

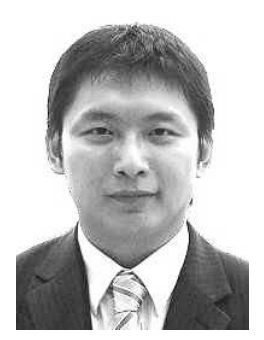

Chih-Liang Chen was born in Tainan, Taiwan. He received the B.S.E.E. degree from National Chung Hsing University, Taiwan, in 2005. He is currently a Ph.D candidate in the Department of Electronics Engineering and Institute of Electronics in National Chiao Tung University. His research interests include digital signal processing, synchronization, channel estimation and equalization.

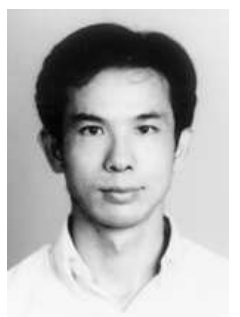

Sau-Gee Chen received his B.S. degree from National Tsing Hua University, Taiwan, in 1978, M.S. degree and $\mathrm{Ph} . \mathrm{D}$. degree in electrical engineering, from the State University of New York at Buffalo, NY, in 1984 and 1988, respectively. During 2003 and 2006, he was director of Institute of Electronics, Department of Electronics Engineering, National Chiao Tung University, Taiwan. Currently, he is a professor at the same organization. His research interests include digital communication, multi-media computing, digital signal processing, and VLSI signal processing. He has published more than 70 conference and journal papers, and holds several US and Taiwan patents. 